J. Clin. Chem. Clin. Biochem.

Vol. 27, 1989, pp. 323-329

(C) 1989 Walter de Gruyter \& Co. Berlin - New York

\title{
External Quality Assessment of Absorbance Measurements on Spectral and Spectral-Line Photometers
}

\author{
By G. Röhle
}

Institut für Klinische Biochemie, Universität Bonn

H. Schlebusch

Universitätsfrauenklinik, Bonn

R. Kruse and W.-J. Geilenkeuser

Institut für Klinische Biochemie, Universität Bonn

(Received June 30/December 9, 1988)

Summary: From 1984-1987, 12 quality control surveys on photometric measurements were carried out in 600-800 laboratories. The participants measured the photometric absorbance of the control samples at 4 wavelengths of the mercury spectrum: $334.1 \mathrm{~nm}, 365.4 \mathrm{~nm}, 404.7 \mathrm{~nm}$ and $546.1 \mathrm{~nm}$.

The medians of the results were without exception lower than the target values, but only very few of them deviated more than $1 \%$. The dispersion of the values did not follow a normal distribution. Two thirds of the values were concentrated within a very small range, while about $10 \%$ lay outside the 2 - to 3 -fold range. It was found that longer wavelengths resulted in a smaller dispersion of readings than shorter ones. Furthermore, precision showed a significant dependency on the absorbance readings of the samples, on the one hand, and on the different photometers, on the other.

\section{Introduction}

Photometers are among the most important measuring instruments for carrying out quantitative determinations in clinical chemistry. Means for the quality control of photometers as well as the results of interlaboratory studies were described in a number of publications (e. g. 1.c. $(1-4)$ ). The objects of the interlaboratory studies, however, were mainly spectrophotometers. A comparison of the readings of a larger number of spectral-line photometers on the one hand, and spectrophotometers, on the other, has not yet been reported.

A carefully maintained photometer should make only a slight contribution to the total error of the analytical results. However, since the error of photometric measurement is usually relatively small, any increase in

this error is not to be expected and remains un-noticed until it becomes quite large. Regular measurement of control samples with a defined absorbance enables the early recognition of photometric error. Such control specimens were sent out by the Department for External Quality Control of the Deutsche Gesellschaft für Klinische Chemie to the laboratories that participated in the quality control surveys for photometers in the years $1984-1987$.

The collected data of the survey consisted of measurements taken in different laboratories with different equipment. In view of these various origins of the data, not every one of the phenomena observed in this study could be interpreted in detail. It was very clear, however, that the majority of measurements showed only slight errors. In contrast, a considerable 
number of results indicated major flaws in the photometers used. Furthermore, it was noticeable that interlaboratory precision in the readings was dependent on the operational wavelength and on the instruments used, i.e. spectral-line photometers and spectrophotometers.

\section{Materials and Methods}

Wavelengths studied

$334.1 \mathrm{~nm} ; 365.4 \mathrm{~nm} ; 404.7$ and $546.1 \mathrm{~nm}$.

\section{Sample material}

The solutions were produced in our own laboratory, then dispensed into $10 \mathrm{ml}$ ampoules. Solutions of the following substances were used: potassium dichromate at $334.1 \mathrm{~nm}$ and 365.4 nm (5) (fig. 1 a), p-nitrophenol at 404.7 (6) (fig. 1 b) and haemiglobin cyanide at $546.1 \mathrm{~nm}(7,8)$ (fig. $1 \mathrm{c}$ ).

\section{Target values}

The target valuies of absorbance (A) were certified results from measurements of the Physikalisch Technische Bundesanstalt, Berlin. The measurements were performed exactly at the wavelenghts given above.

\section{Quality control surveys}

Between 1980 and 1983 two surveys per year were offered, while three surveys per year were offered from 1984 onwards. In 1987 the average number of participating laboratories was 700 per survey. Of these, around $15 \%$ used a spectrophotometer, the rest a spectral-line photometer with a mercury lamp. Each participant received a total of 6 ampoules for each survey: 2 each with solutions of potassium dichromate, $p$-nitrophenol and haemiglobin cyanide. Measurements were to be performed with water as blank. Each participant received at least 4 new samples of identical material. The results were collected and evaluated by the director of the survey.

\section{Construction of the dispersion profiles of figures} $2 a-d$

The regression curves were calculated on the basis of the relative differences between the medians and the respective 25 th and 75 th percentiles of the quality control surveys (9). Collective readings from users of the same type of photometer have been kept separate (in the figures marked by a pair of dots under and over the abscissa). They were weighted according to the respective number of individual readings, and accounted for in the calculation of the regression curve (10).

\section{Data to figures $4 \mathrm{a}-\mathrm{c}$}

Instrument group 1 represents equivalent spectral-line photometers from a single manufacturer and makes up the largest sub-group. Instrument group 2 represents equivalent spectralline photometers of another manufacturer and makes up the second largest sub-group. Instrument group 3 represents equivalent spectrophotometers of still another manufacturer; it is the largest sub-group for this kind of photometer.
The small number of results in instrument group 3 was the reason that quality control surveys $3 / 84,3 / 85$ and $3 / 86$ were combined in figures $4 \mathrm{a}-\mathrm{c}$. In these three surveys, identical material was used for all the samples.

For better comparison of the figures, the histograms were normalized to the same area.

\section{Definitions}

\section{.1}

The following terms, among others, were used: scattered radiation, stray light, ambient radiation. Since the use of these terms in the relevant literature is not uniform, they are defined as follows for present purposes.

Scattered radiation is that part of the radiation which, on the way from the monochromator to the radiation receiver, is dispersed without a change in wavelength. In case there is a difference in the amount of radiation scattered by e.g. a somewhat different placement of the cuvette (water blank, control sample resp.) in the holder, this can lead to an increase or decrease in the readings for the control samples; scattered radiation, which gets past the sample and to the receiver, lowers the absorbance readings.

Stray light is that part of the radiation whose wavelength differs from that selected for the photometric measurement. With nonmonochromatic radiation sources it is hardly possible to eliminate stray light completely. For physical and technical reasons (e. g. finite band widths, the limited performance of filters), the intensity of this stray light generated by photometers is, in general, more easily noticeable at shorter than at longer wavelengths. When stray light absorption is less than that of the selected measurement radiation, it results in a lowered rate of absorbance.

Ambient radiation: penetration of light from the surroundings into the photometer is in principle avoidable. If it nevertheless occurs, it will also lead to a lowered absorbance reading for the sample.

\section{Results and Discussion}

\section{Choice of the control material}

There were two main considerations for the selection of the specimen material.

- An indispensable requirement was that the absorbance qualities of the samples be stable over a fairly long period.

- It then seemed reasonable to test the reliability of the photometer with chemical compounds that are measured routinely in clinical chemical laboratories.

Both requirements were fulfilled by $p$-nitrophenol and haemiglobin cyanide solutions for the frequently used wavelengths of the mercury emission spectrum at $404.7 \mathrm{~nm}$ and $546.1 \mathrm{~nm}$, respectively. NAD̈H , the concentration of which serves as an object of photometric measurement at wavelengths of around 340 $\mathrm{nm}$ in many analysis methods, is not stable enough. Therefore, for testing absorbance at $334.1 \mathrm{~nm}$ and $365.4 \mathrm{~nm}$, solutions of potassium dichromate were preferred. 
Gray solutions, if ever available in optimum quality, and - with reservations - picrate solutions (11), both of which show a relatively even absorbance in the relevant spectral range, have, on the one hand, the advantage that absorbance can be tested for almost any wavelength. But on the other hand, it is this even absorption that prevents the detection of straylight error, since radiation of all wavelengths is more or less absorbed and not differentiated.

The relatively narrow absorbance bands of potassium dichromate, $p$-nitrophenol and - to a lesser degree - haemiglobin cyanide in the respective wavelength ranges of 334.1, 365.4, 404.7 and $546.1 \mathrm{~nm}$ (fig. 1) respond more sensitively to spurious radiation. In spectrophotometers this increases the possibility of recognizing an error in the wavelength setting or, in spectral-line photometers, a shift in the effective centres of the wavelengths possibly due to aging filters.

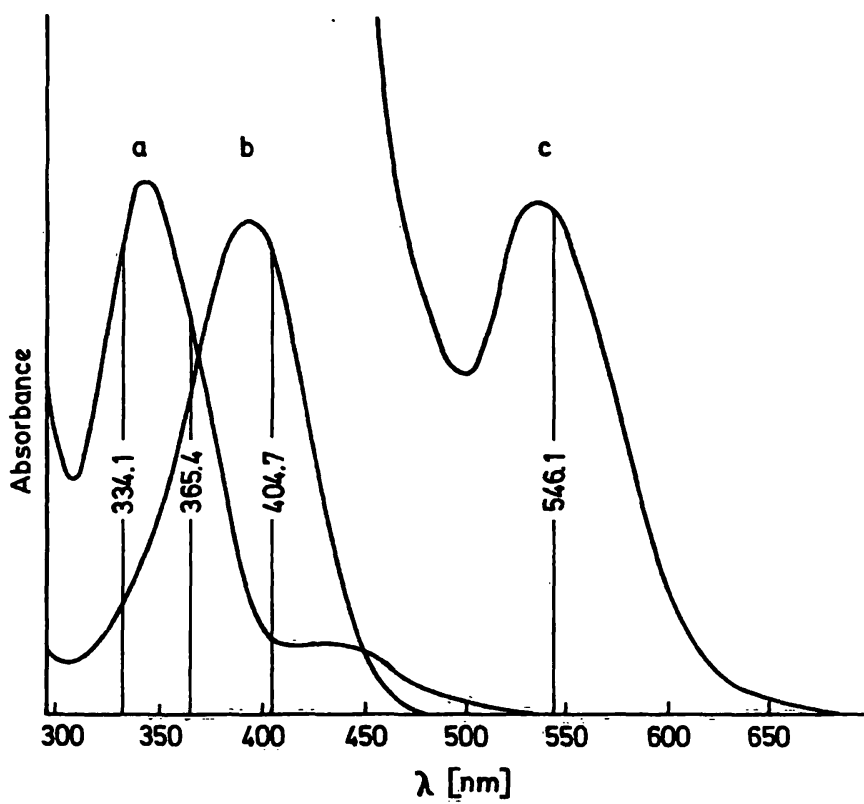

Fig. 1. Absorption spectra of solutions of

a) potassium dichromate

b) $p$-nitrophenol

c) haemiglobin cyanide

\section{Accuraçy}

In general, there were only slight differences between the medians of the survey results and the target values determined at the same wavelengths, mostly less than $-1 \%$. Comparable deviations were observed by other authors $(3,4)$ reporting survey results on spectrophotometers only. The fact that all target values lay above the medians (fig. 2) is probably explained by the larger contribution from scattered radiation and stray light in routine measurements than under the optimal conditions of target value determination.
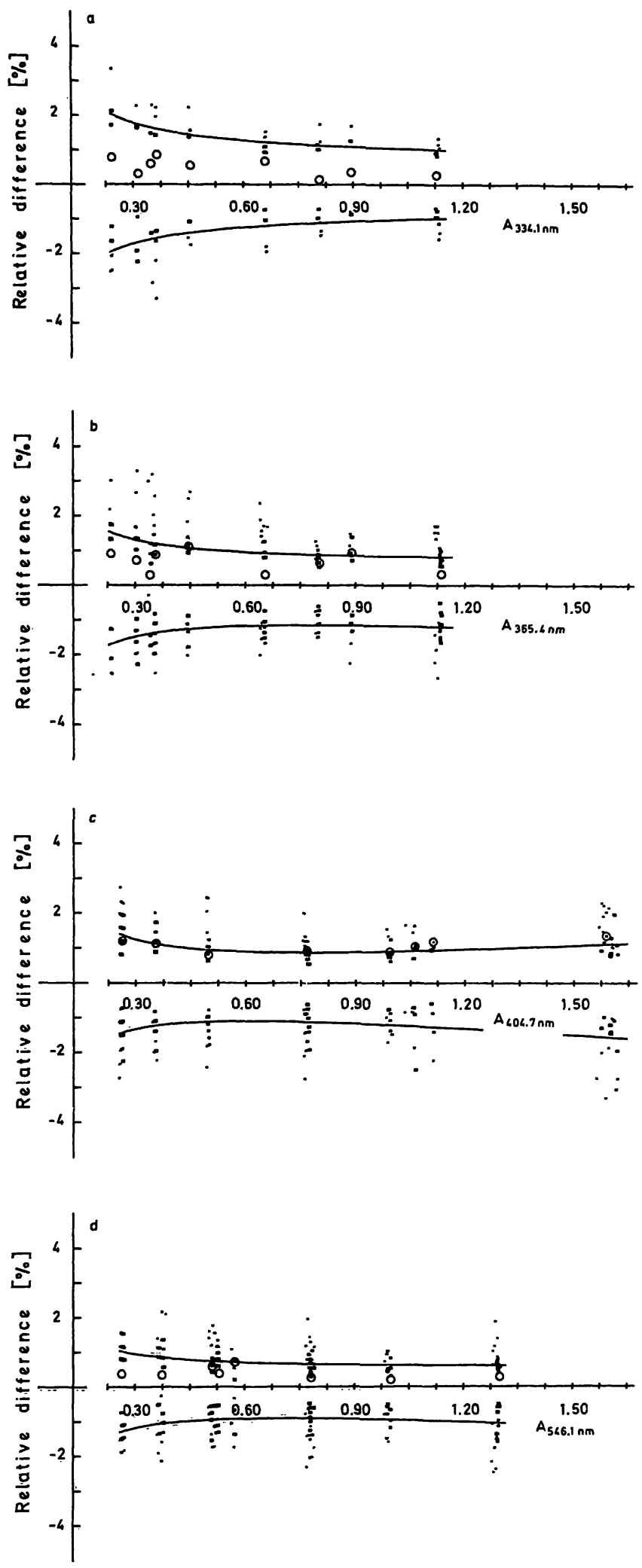

Fig. 2. Dispersion profiles for photometric absorbance (A) measurements at 4 wavelengths $(\lambda)$ compiled from the relative differences between the medians (abscissa) and, respectively, the 25 th (lower curve) and 75 th percentiles (upper curve) supplied by the subcollectives of 24 survey results.
a) $=334.1 \mathrm{~nm}$
b) $=365.4 \mathrm{~nm}$
c) $=404.7 \mathrm{~nm}$
d) $=546.1 \mathrm{~nm}$
0 = location of target value relative to the correspond- ing median




\section{Interlaboratory precision}

Along with the unavoidable random errors which influence all measurements to a greater or lesser degree, systematic errors in individual laboratories can effect interlaboratory precision. An example from the survey test $3 / 1984$, chosen because it offers the largest number of readings, shows that in the distribution of the results from measurements at $404.7 \mathrm{~nm}$ and without considering differences in equipment, both kinds of error played only a very small role in most laboratories (fig. $3 \mathrm{a}$ ).

The range from median-minus-standard deviation to median-plus-standard deviation comprised $0.044 \mathrm{ab}-$ sorbance units. If there had been a normal distribution, $68 \%$ of the readings would have been within the limits of this range. But as the difference between the 84 th and 16 th percentile shows, $68 \%$ were in the very much narrower range of only 0.024 absorbance units. These collective readings had then - along with all the other quality control survey results studied - a much higher precision than the standard deviation stated. The cause of this misleadingly large standard deviation was a comparatively small, but still not negligible number of results with very high deviation from the median.

A further basis for appraising interlaboratory precision was suggested by the Physikalisch Technische Bundesanstalt, i.e. that tolerance limits for photometric measurements (12) be constructed as follows: on the basis of the target value $\left(A_{t}\right)$, the values for the lower and upper limit $\left(A_{1}\right.$ and $\left.A_{u}\right)$ are to be set at:

$$
\begin{aligned}
& A_{1}=0.97 \cdot A_{t}-0.01 \\
& A_{u}=1.03 \cdot A_{t}+0.01
\end{aligned}
$$
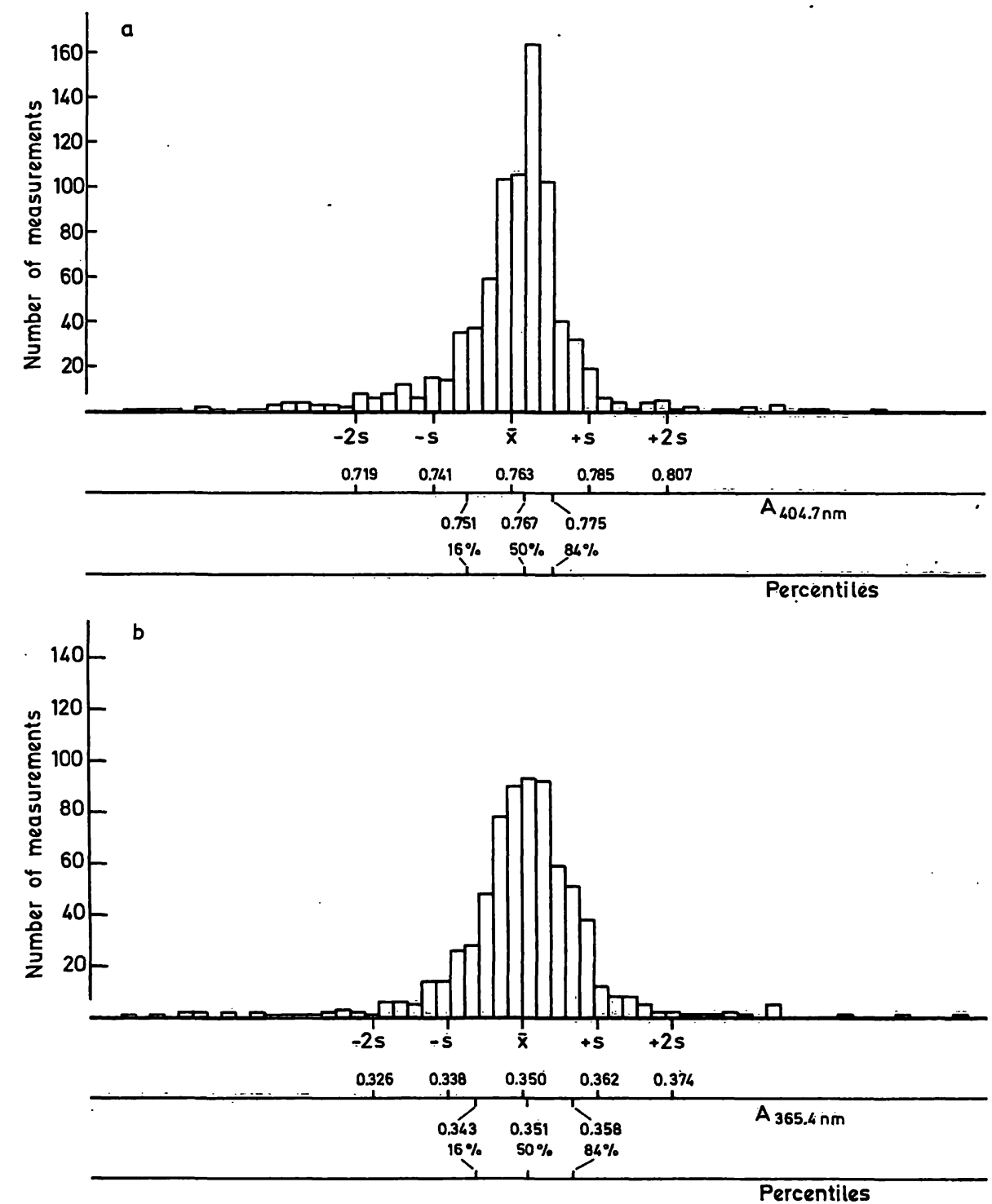

Fig. 3. Frequencies of absorbance readings in quality control survey $3 / 84$

a) at $404.7 \mathrm{~nm}$ for specimen lot No. $1550(\mathrm{n}=828)$

b) at $365.4 \mathrm{~nm}$ for specimen lot No. $2349(\mathrm{n}=721)$, with the means $(\overline{\mathrm{x}})$, standard deviations $(\mathrm{s})$ and percentiles. $\mathrm{n}=$ number of readings 
In the example shown in figure $3 \mathrm{a}$, the target value for the sample material was 0.774 . Lower and upper limits would have therefore been, respectively, 0.741 and 0.807 absorbance units. The values of the previously defined $68 \%$ of the participants were concentrated in hardly more than one third of this generous range. However, of 828 values, 73 were below and 14 above the limits.

Comparable findings resulted from a second example of the same survey with other values for wavelength and absorbance (fig. 3 b).

The study of factors that possibly influence the results of interlaboratory precision provided a variety of interrelations. The dispersion of the values were dependent on

- the value of the absorbance

- the wavelength

- the photometer used

The regression curves in figure 2, which have been calculated on the basis of the weighted 25 th or 75 th percentile of 24 surveys, showed as their most striking feature that below $A=0.5$ there was an increase in relative dispersion of the readings with a decrease in absorbance. This corresponded qualitatively to the theoretical calculations of errors in photometric measurements (13). According to these calculations, absorbance values above about 1.2 , again show an increase in relative dispersion of the measurements to be expected, this time with increasing absorbance values. At a wavelength of $404.7 \mathrm{~nm}$, at which an absorbance of up to 1.6 was measured in the surveys, this theoretically substantiated trend is clearly apparent (fig. 2c).

Like the differences between medians and target values, the dispersions in figure 2 show that the tendency to negative is higher than to positive deviation. As regards medians, the regression curves for the 25th percentile, except at a wavelength of 334.1 (fig. 2 a), were at a greater distance from the abscissa than those for the 75th percentile. As regards target values, there was greater deviation of the 25th percentiles in all instances. These findings can also be explained by the fact that the most important sources of error in photometry (i.e. stray, scattered and ambient radiation) lead more frequently to lower, rather than higher, absorbance values.

The dispersion in readings varied according to the individual wavelength. At $\mathrm{A}=0.60$ (at about the middle of the absorbance range under observation) the sum of the relative differences between the medians and the regression curves of, first, the 75th and, second, the 25 th percentile were: $2.6 \%$ for $334.1 \mathrm{~nm}$, $2.1 \%$ for $365.4 \mathrm{~nm}, 2.0 \%$ for $404.7 \mathrm{~nm}$ and $1.6 \%$ for $546.1 \mathrm{~nm}$ (fig. $2 \mathrm{a}, \mathrm{b}, \mathrm{c}, \mathrm{d}$ ).

Thus, the dispersion was more noticeable at shorter wavelengths. Considering the fact that stray light is generated more in shortwave than in longwave light, the observed dependency on wavelength would be one, but not the only, explanation.

The factors that cause this phenomenon are, without doubt, in type and range different from instrument to instrument, and the influence of other instrumental errors that lower or raise absorbance values cannot be discounted. Fundamentally, however, the results accord with the general experience that measurements at shorter wavelengths are more influenced by stray light than at longer ones. Furthermore, the characteristic for the absorbance spectrum of haemiglobin cyanide means that with measurements at $546.1 \mathrm{~nm}$, greater absorbance of stray light may occur than does with the other two solutions used for shorter wavelengths (fig. 1). This explains the very slight dispersion of the readings at $546.1 \mathrm{~nm}$.

Photometers differ according to function and construction. It was reasonable to assume that these differences would be manifested in the readings. This proved to be the case, after the results (shown in part in figure 3) were divided into groups of instruments (see Materials and Methods). In instrument group 1 (spectral-line photometers), the range from the 84th to the 16 th percentile extended only over $0.019 \mathrm{ab}-$ sorbance units (fig. 4 a). For the $68 \%$ of the values that lay in this range, the maximum negative deviation from the median was -0.010 and the maximum positive deviation +0.009 units. Instrument group 2 (spectral-line photometers) showed a corresponding range of 0.031 absorbance units with deviations of -0.021 and +0.010 (fig. 4 b). This was even greater for spectrophotometers of instrument group 3 in the 84th to 16 th percentile range, with 0.046 absorbance units and limits that showed a median difference of -0.031 and +0.015 (fig. $4 \mathrm{c}$ ). The coefficient of variation $(3.3 \%)$ was in the same order as that which Jansen et al. calculated (3.5\%) from measurements on the almost identical control material performed with spectrophotometers (4). Larger dispersion in the measurement results of spectrophotometers is probably due to an error in adjusting the wavelength, an error that is obviously not possible with spectral-line photometers. 

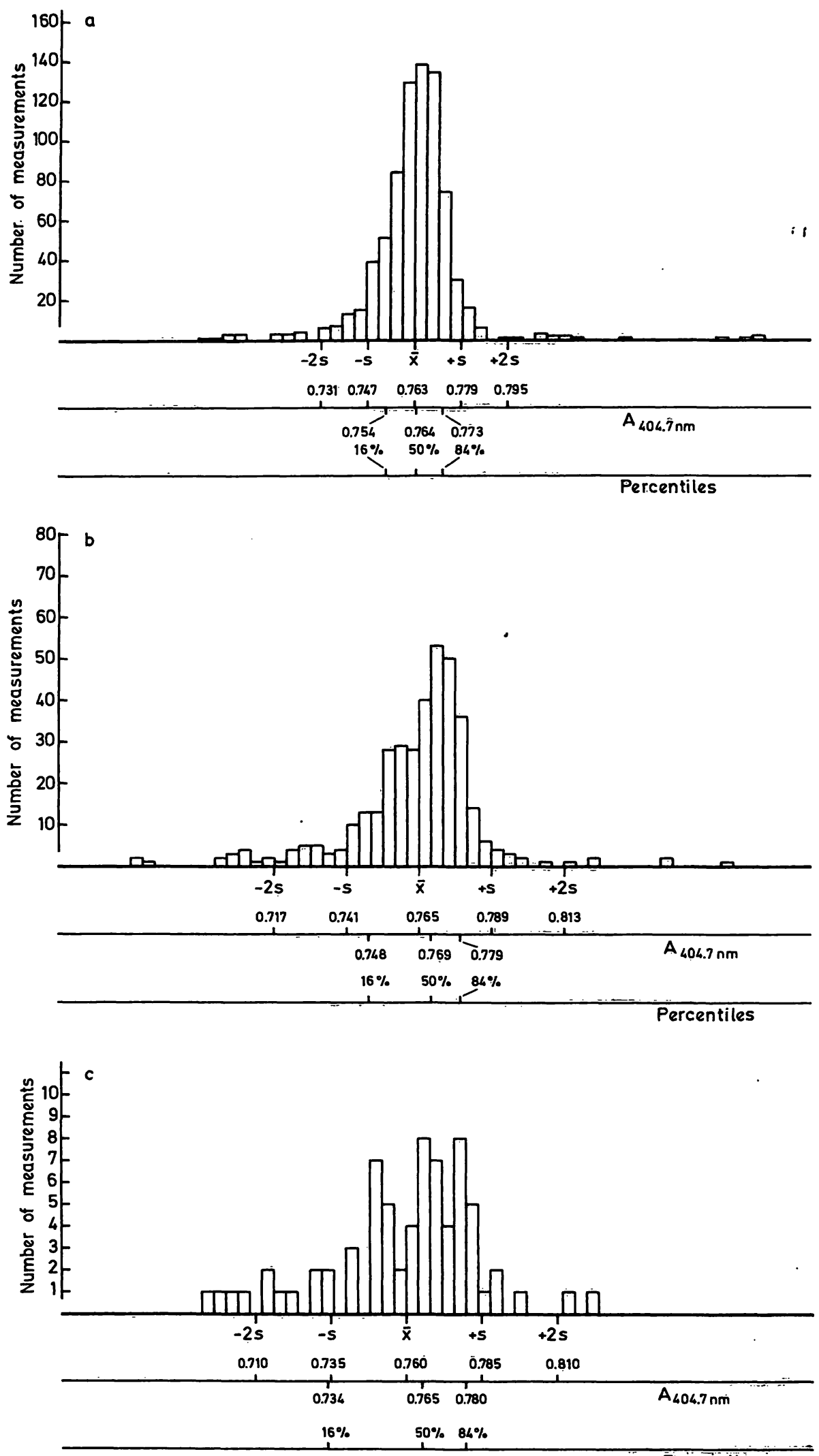

Percentiles

Fig. 4. Frequencies of absorbance readings in surveys 3/84, 3/85, 3/86 for the specimen lot No. 1550 at $404.7 \mathrm{~nm}$ carried out with

a) spectral-line photometers of instrument group $1(n=775)$

b) spectral-line photometers of instrument group $2(n=375)$

c) spectrophotometers of instrument group $3(n=71)$, with the means $(\overline{\mathbf{x}})$ and standard deviations $(s)$. $n=$ number of readings 


\section{Conclusions}

The results from the quality control surveys on photometry show that most of the participating laboratories can carry out very precise photometric measurements. The most reliable measurements are obtained from properly maintained spectral-line photometers. Differences due to inherent variations in the manufacturing design cannot be discounted.

With spectrophotometers, the advantage of a free choice of wavelength means accepting a somewhat greater dispersion of measurement.

The proportion of photometers with readings that point to major flaws is too high: more than $10 \%$ among participants, all of whom were voluntary. It

\section{References}

1. Burke, R. W., Deardorff, E. R. \& Menis, O. (1972) Liquid absorbance standards. J. Res. Nat. Bur. Stand. (U.S.) $76 \mathrm{~A}$ (Phys. and Chem.), 469-482.

2. Rand, R. N. (1969) Practical spectrophotometric standards. Clin. Chem. 15, 839-863.

3. Vanderlinde, R. E., Richards, A. H. \& Kowalski, P. (1975) Linearity and accuracy of ultraviolet and visible wavelength photometers: An interlaboratory survey. Clin. Chim. Acta $61,39-46$.

4. Jansen, A. P., van Kampen, E. J., Steigstra, H., van der Ploeg, P. H. W. \& Zwart, A. (1986) Simultaneous spectrophotometric calibration of wavelength and absorbance in an interlaboratory survey using holmium oxide $\left(\mathrm{Ho}_{2} \mathrm{O}_{3}\right)$ in perchloric acid as reference, compared with $p$-nitrophenol and cobaltous sulphate solutions (1978-1984). J. Clin. Chem. Clin. Biochem. 24, 141-146.

5. Burke, R. W. \& Mavrodineanu, R. (1977) Certification and use of acidic potassium dichromate solutions as an ultraviolet absorbance standard - SRB 935. NBS Spec. Publ. 260-54.

6. Bowers, G. N. \& McComb, R. B. (1975) Measurement of total alkaline phosphatase activity in human serum. Clin. Chem. 21, 1988-1995.

7. Van Kampen, E. J. \& Zijlstra, W. G. (1983) Spectrophotometry of hemoglobin and hemoglobin derivatives. Adv. Clin. Chem. 23, $199=257$. is to be feared that this is even higher in laboratories that do not participate in surveys.

From the large number of almost faultless results, which show the degree of interlaboratory precision that is achievable, one can assume that the number of values with incomparably large deviations could be greatly reduced if all laboratories would carry out regular photometer checks.

\section{Acknowledgement}

We would like to thank Prof. Dr. G. Nöldecke, director of the Physikalisches Institut der Universität Bonn, as well as Dr. Th. Bronder and Dr. H. Rinneberg, Physikalisch-Technische Bundesanstalt, Berlin for in-depth discussions and important suggestions. We also thank Ms. J. Haunschild, Institut für Klinische Biochemie der Universität Bonn, for translating the manuscript.

8. Hallmann, L. (1980) Klinische Chemie und Mikroskopie, 11th ed., pp. 70-71. Georg Thieme Verlag Stuttgart, New York.

9. Röhle, G. \& Voigt, U. (1986) Concept for the evaluation of analytical results in clinical chemistry. Part I: Concentration-dependent dispersion profiles of survey results. Commun. Lab. Med. 2, 81-85.

10. Röhle, G., Voigt, U. \& Siekmann, L. (1986) Concept for the evaluation of analytical results in clinical chemistry. Part II: The accumulated distribution-free dispersion parameter and its application. Commun. Lab. Med. 2,131138.

11. v. Klein-Wiesenberg, A., Merten, R. \& Reinauer, H. (1987) in Qualitätssicherung im Medizinischen Laboratorium (v. Boroviczény, K.-G., Merten, R. \& Merten, U., eds.) pp. $872-885$, Springer-Verlag. Berlin, Heidelberg, New York, London, Paris, Tokyo.

12. In Qualitätssicherung im Medizinischen Laboratorium (v. Boroviczény, K.-G., Merten, R. \& Merten, U., eds.) pp. 972-974, Springer-Verlag. Berlin, Heidelberg, New York, London, Paris, Tokyo.

13. Kortüm, G. (1962) Kolorimetrie, Photometrie und Spektrometrie, Vol. II, 4th ed., pp. 247-251, Springer-Verlag. Berlin, Göttingen, Heidelberg.

Priv. Doz. Dr. G. Röhle

Institut für Klinische Biochemie

Sigmund-Freud-Straße 25

D-5300 Bonn 1 
